\title{
Facades inspection with infrared thermography: cracks evaluation
}

\author{
Elton Bauer $^{1}$ (1) $\cdot$ Elier Pavón $^{2} \cdot$ Elias Oliveira $^{1} \cdot$ Claudio H. F. Pereira $^{1}$
}

Received: 24 June 2016/Accepted: 19 August 2016/Published online: 12 September 2016

(C) Springer International Publishing Switzerland 2016

\begin{abstract}
Infrared thermography is an evaluation technique that helps to identify anomalies and can provide information on their incidence and severity. The aim of this study is to apply quantitative thermography to study a case of severe cracks in facade analyzing the evolution of temperatures and Delta-T. The temperature evolution was studied over a day. There was sun incidence occurring during part of the day, allowing to calculate and assess the evolution of the associated temperatures. Thermograms were acquired hourly during the study period. The highest temperatures were observed at 12:00 noon, and the larger Delta-T between 10:00 and 12:00. There were differences between the Delta- $\mathrm{T}$ profiles among the three regions studied. The best time for analysis is when Delta-T is higher. Cracks with higher Delta-T were considered more degraded, and generalized branched cracks did not allow a comparative conclusion.
\end{abstract}

Keywords Infrared thermography $\cdot$ Facade $\cdot$ Crack · Delta-T

Elton Bauer

elbauerlem@gmail.com

1 Post-Graduate Program in Structures and Civil Construction, Civil and Environmental Engineering Department, University of Brasília, Campus Universitário Darcy Ribeiro-Asa Norte, Brasília, DF, Brazil

2 Centro das Ciências Exatas e das Tecnologias Post-Graduate Program in Structures and Civil Construction - UnB, Universidade Federal do Oeste da Bahia-UnB, Campus Reitor Edgar Santos, Barreiras, Bahia, Brazil

\section{Introduction}

Infrared thermography is a technique used to study the pathology and anomalies of buildings. This technique applied to the identification and mapping of faults allows a new inspection approach that enables classifying the damage areas regarding the occurrence intensity and severity of anomalies.

Thermography is a non-destructive technique that provides a thermogram, a thermal image of the target object surface, which in this case is a region of the facade. This inspection, made without physical contact, can reach distances of tens of meters and causes no damage to the element. The result is given in real time, i.e., the temperatures observed in the thermographic camera are those occurring on the target object at the time [1].

The thermogram of a facade allows analyzing the surface temperatures of the elements and materials that compose it. Obviously, temperatures are a result of the action of various factors, including the action of climatic agents such as the incidence of the sun and ambient temperature. Elements in thermal or hygroscopic equilibrium with the environment are difficult to study in thermography since significant differences in surface temperature become more difficult to identify [2]. There must be a heat flux from the external environment to the facade (direct) or from the facade to the environment (inverse) to identify the anomalies. These fluxes occur naturally by the action of climatic agents on the facade. The defects or anomalies cause disturbances to the heat flux, leading to a distribution of different temperatures on the surface of the facade or element, which is captured by the thermogram. The main difficulty, in thermographic inspection, is to identify at which heat flux condition the defects are visualized, and how they manifest themselves [3, 4]. 
Different types of inspection may be used when applying the thermography. If the heat flux used to study the thermography has a natural origin, for example, derived from sunlight on the facade, this study technique is named passive thermography. If the heat flux is induced on the element by heating, vibration, ultrasound, among others, the technique is known as active thermography $[5,6]$. Thermography can also be considered qualitative when it is based only on the identification of hot and cold spots in the thermographic image. In this case, the analysis consists of comparing the images with standards, this type of study is usually used in restorations and diagnosis of historic buildings [7]. Otherwise, the quantitative thermography is used when the objective is to classify the importance of the defect, and the greatest concern is the accuracy of the obtained temperatures, as well as using the proper techniques to obtain thermograms and analyze the relevant information [8]. This approach requires accurate measurement and assessment of the material parameters such as emissivity and reflected apparent temperature. These parameters should be properly introduced in the thermogram analysis software to be able to work quantitatively with the required accuracy [9-11].

In the quantitative analysis, Delta- $T$ is the initial parameter, corresponding to the temperature difference between the defective area and the defect-free area. Comparatively, for the same exposure conditions, a greater Delta-T may indicate a more severe condition for the anomalies [12]. Bauer et al. [4] correlated the depth of cracks with the Delta- $T$ values measured in laboratory heating cycles and reported that higher Delta-T values are associated with deeper cracks. Thus, the thermogram obtained from the quantitative thermography study can indicate the severity degree of the anomaly.

The cracks of the facade may be due to anomalies in the coating material, or associated with the substrate elements (masonry) [13]. The diagnosis needs to establish the causes of cracks, identify the existing typology and the degree of damage. The use of thermography to study cracks, detachment and humidity are still being performed on an experimental basis, even the field studies. The studies have shown a strong dependence on the variations of surface temperature and sun incidence, with no agreement about the criteria regarding the best time to make the thermographic inspection (day or night) $[15,16]$. It can be said, obviously, that the weather conditions in the country where the studies are being performed are an important variable in the processes and results since they involve differentiations of heat flux for each study [4, 14].

Few field and laboratory studies have been made regarding the degradation of facades by cracking. Most often, thermography has been used to observe the location of cracks [4], and not as quantitative thermography, i.e., without evaluating the severity of the anomaly.

The aim of this study is to apply quantitative thermography to study a case of severe cracks in a building facade. The temperature evolution throughout the day is investigated using thermography. The incidence of the sun during part of the studied period allowed calculating and evaluating the evolution of the associated temperatures and classifying the severity of the anomalies.

\section{Development of experimental methodology}

The experimental study was performed on the facade of a building in Brasilia, Brazil, characterized by the existence of cracking. The facade, coated in mortar, has two types of cracking, linear and branched. In the mapping, three study regions were considered: $\mathrm{A}, \mathrm{B}$, and $\mathrm{C}$. Figure 1 shows the spots chosen for the thermographic study, three spots were defined in region $A$, three in region $B$, and two in region $C$. The spots A1, A2 and A3 of region A are located on the main crack with linear characteristics. B1 and B2 of the area $\mathrm{B}$ follow a linear crack while $\mathrm{B} 3$ represents a branched crack. $\mathrm{C} 1$ and $\mathrm{C} 2$ of area $\mathrm{C}$ refer to a region of generalized branched cracks.

The thermographic monitoring consisted of studying the thermograms and obtaining the temperature at specific spots in the cases of linear cracks (A1, A2, A3, B1, and $\mathrm{B} 2$ ), and the average temperature of the cracked area in the case of branched cracking (B3, C1, C2), as shown in Fig. 1a.

The evolution of the thermal behavior of the cracks is evaluated by comparing the temperature of the defective region (TD) with the temperature of defect-free region (TND). Thus, defect-free spots, where TND was determined initially in the thermogram, were kept fixed throughout the study.

The research procedure consists of thermography monitoring of the facade in the studied region employing passive thermography. The east facade was exposed to the sun in the morning, from 7:00 to 12:00 noon. The study was conducted in July, period with no rainfall and clear skies in Brasilia. The temperature evolution throughout the day was defined by performing a hygrothermal simulation of the facade using the Wuf ${ }^{\circledR}$ Pro 5.3. Data on existing materials and a typical meteorological year (TMY) file specific to the climate of Brasilia were used for the simulation. The absorbance of 0.3 was considered.

The study consisted of obtaining thermograms every hour during the inspection time, which started at 8:00 a.m. and ended at 6:00 p.m. The sunlight period was observed in the morning (between 8 a.m. and 12 noon), and the period without sunlight (shading) after 12 noon. 
Fig. 1 a Facade: definition of regions and analyzed spots; b thermogram obtained for a determined inspection time (8:00) (a)

(b)

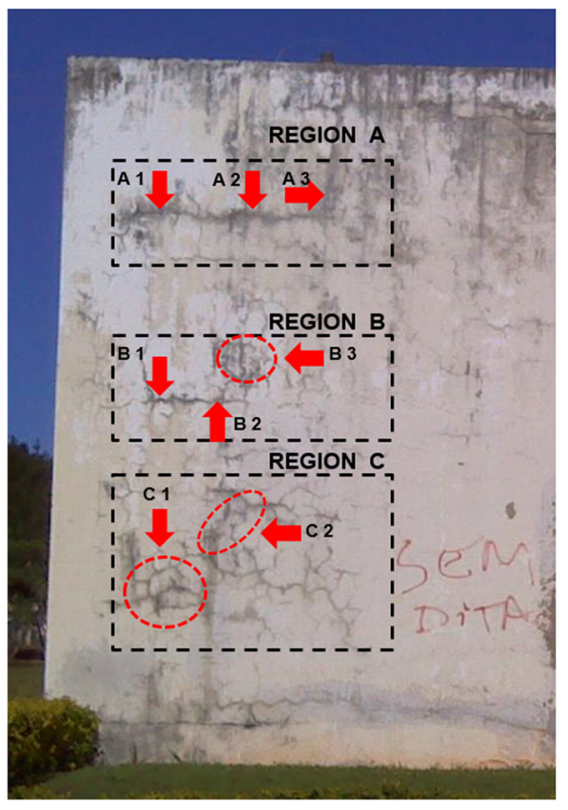

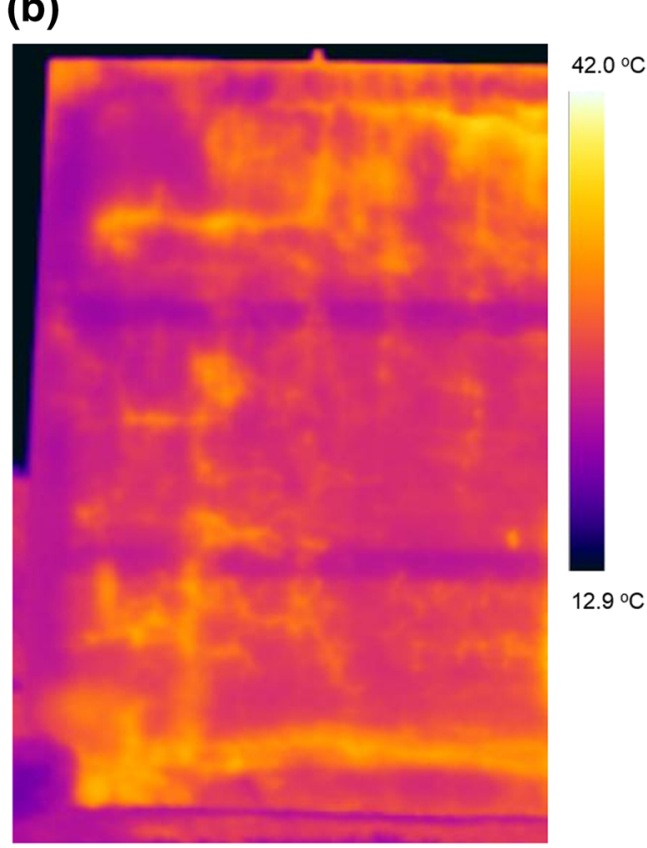

All images were made in sunlight. All thermograms were acquired at a fixed distance of $18.5 \mathrm{~m}$ from the facade. The reflected apparent temperature (TAR) was determined for each time, using the method of corrugated aluminum sheet, as defined by ASTM E1862 [11]. The emissivity was determined following the standard black tape method, as described by ASTM 1933 [10]. Ambient temperature (T) and relative humidity (RH) were measured at each time using a MeterLink MO297 thermohygrometer. The infrared camera used was the Flir T400 model, with -20 to $120{ }^{\circ} \mathrm{C}$ temperature range, $2 \%$ accuracy, 7.5 to 13 micrometers spectral range, $320 \times 240$ pixels resolution, $25^{\circ}$ lenses, and $1.36 \mathrm{mrad}$ IFOV. The image analysis was performed using the free version Flir QuickReport 1.2 software. The thermograms were acquired routinely following the steps:

- The corrugated aluminum sheet was attached to the surface of the facade, and the image was obtained at a distance of $2.0 \mathrm{~m}$. This step is necessary to obtain the TAR, as this parameter is an input data requested by the infrared camera.

- Positioning of the thermographer at the point of image acquisition (distant $18.5 \mathrm{~m}$ from the facade), and determining the relative humidity $(\mathrm{RH})$ and ambient temperature $(\mathrm{T})$. Inserting the data in the camera software.

- Adjustment of the thermal focus and acquisition of three consecutive images.

- Subsequent analysis of the images in Flir QuickReport 1.2 to obtain the temperatures on the studied spots.

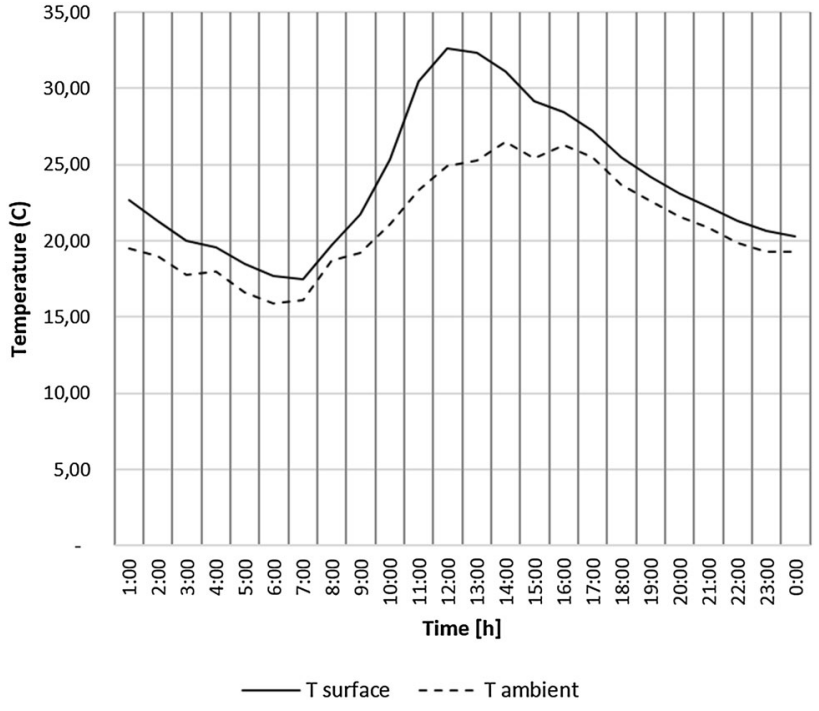

Fig. 2 Evolution of surface and ambient temperatures (hygrothermal simulation)

\section{Results and discussion}

The use of passive thermography to study cracks requires the knowledge on the evolution of surface and environment temperatures to be able to understand the obtained results. Figure 2 shows the results observed on the hygrothermal simulation. The incidence of solar radiation on the facade starts at 7:00 am, which is apparent by the increasing surface and ambient temperatures. This harsh sunlight hits the studied facade until 12:00 noon. It can be stated that during this period the facade is heating up, or heat flux is 
direct (from outside to inside). After 12.00 noon, when the sun ceases to hit the facade, the surface temperature starts to drop, approaching the ambient temperature at 12:00 midnight (around $20{ }^{\circ} \mathrm{C}$ ).

The investigation of faults or anomalies by thermography is associated with the disturbance that such defects cause in the heat fluxe in the facade (from outside to inside in this case), and how this disturbance changes the surface temperature, obtained in a thermogram. Thus, an internal or surface discontinuity such as a void, detachment or a cracking process can be detected in the thermograms $[12,17]$. The critical question is to be able to identify which of the observed patterns indicate the presence and extent of cracking. Figure 3a shows the first thermogram obtained in the inspection, where the cracks can be identified as longitudinal bands of higher temperature. As already identified in the study areas (Fig. 1a), it is easy to observe the studied regions and the defined cracks in the thermogram. The thermogram captured at 10:00 a.m. here used as reference, it shows the facade in the process of strong heating up $\left(25^{\circ} \mathrm{C}\right)$, and it is easily seen that TD is significantly higher (Fig. 3b). The Delta-T (between TD and TND) is significantly greater, which increases the thermal contrast, facilitating the identification of defects. Now, it becomes difficult to identify the defects accurately in the thermogram obtained at 3:00 p.m. (Fig. 3c). In this situation, the facade temperature is dropping due to the absence of direct sunlight and, therefore, the heat flux decreases and may even be in the inverse direction (inverse fluxe). It is important to note the great dependence of the thermographic observation on the magnitude and direction of heat flux [17]. Surely, there are situations where the defect visibility changes, so it is very important that the thermographer knows the main variables that define these conditions [1].

The quantitative study of the temperature is important from the viewpoint of identifying the defects and their extension, and a preliminary inference of their severity. It should be remembered that thermography is proposed as a distance diagnostic technique, i.e., under conditions in which there is no physical access to the defects [3]. Two evaluations are currently applied, one being the evolution of TD and TND, and another, the evolution of Delta-T (between TD and TND). Figure 4 shows the three spots observed in region A (Fig. 1a). First, a temperature increase is observed during the period of solar irradiation (Fig. 4a), for both TD and TND. The temperatures (for A1, $\mathrm{A} 2$, and $\mathrm{A} 3$ ) were very close in the defect region (TD) throughout the experiment (in the heating and cooling phases), with a maximum value at 12:00 noon, as suggested by the simulation (Fig. 2). In the defect-free region, the temperature in the $\mathrm{A} 3$ spot was higher than in the other two spots, showing a different cracking pattern in that spot (A3). A maximum TND value was observed at 12:00 noon. As the facade starts to cool down (after 12:00 noon), there is a trend for all temperatures to converge, which occurs at 4:00 p.m. in the thermogram when both TD and the TND of the three studied spots approach $25{ }^{\circ} \mathrm{C}$. For maximum Delta-T (Fig. 4b), all three spots are very clear, with values of $9.4,8.7$, and $6.6{ }^{\circ} \mathrm{C}$, for $\mathrm{A} 1, \mathrm{~A} 2$, and $\mathrm{A} 3$, respectively. Regarding thermographic analysis, these values are representative of anomalies [6, 4]. Delta-T is maximum at 9:00 a.m. and 10:00 a.m., dropping heavily until 2:00 p.m., and stabilizing close to $1.0{ }^{\circ} \mathrm{C}$. Therefore, it can be said that the (a)

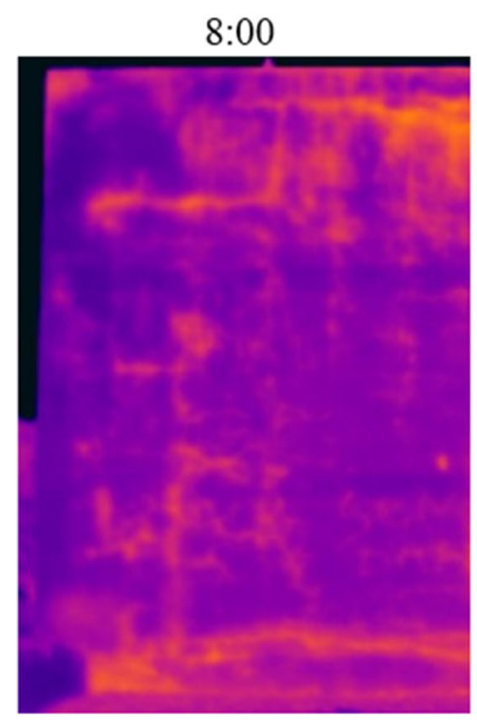

(b)

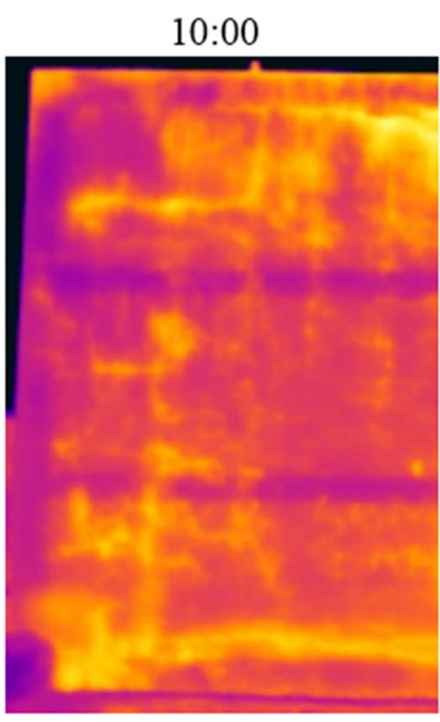

(c)

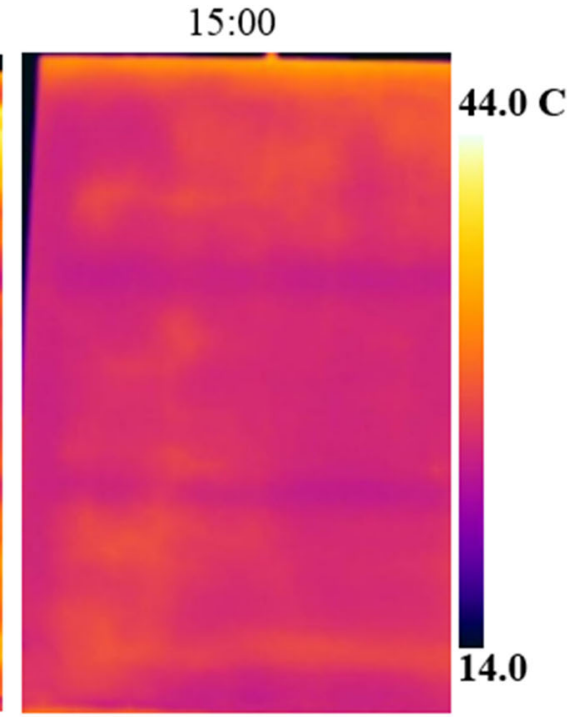

Fig. 3 Thermograms captured during the inspection. a At 8:00 a.m.; b at 10:00 a.m.; c at 3:00 p.m. 


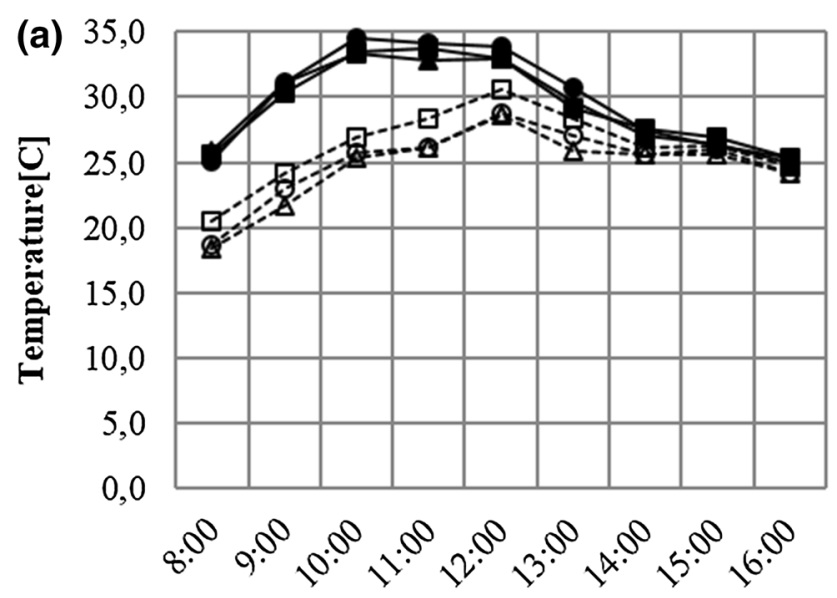

Time [h]

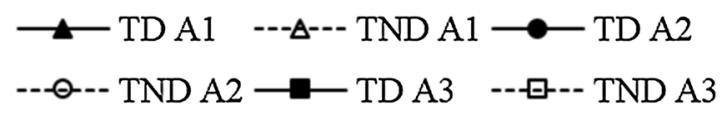

Fig. 4 Region A: a TD and TND evolution; b Delta-T evolution

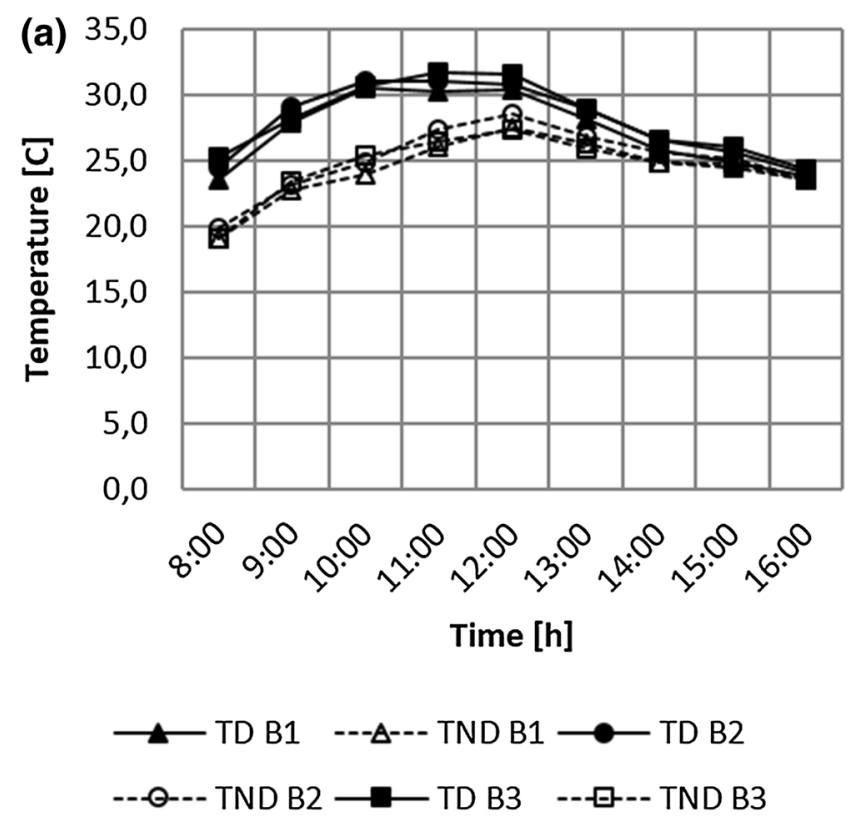

Fig. 5 Region B: a TD and TND evolution; b Delta-T evolution

anomalies are best visualized in the times of maximum Delta-T.

Figure 5 shows the thermogram for region $\mathrm{B}$; B1 and B2 spots are linear cracks. The B3 crack is a system of branched cracks. The TD evolution shows a similar behavior for the three cracks, peaking between 11:00 and 12:00 noon. TND also grows evenly in the studied cracks, peaking at 12:00 noon. During the cooling, the temperature begins to stabilize at 2:00 p.m. reaching $25{ }^{\circ} \mathrm{C}$ at 4:00 p.m. The Delta-T peak values were also
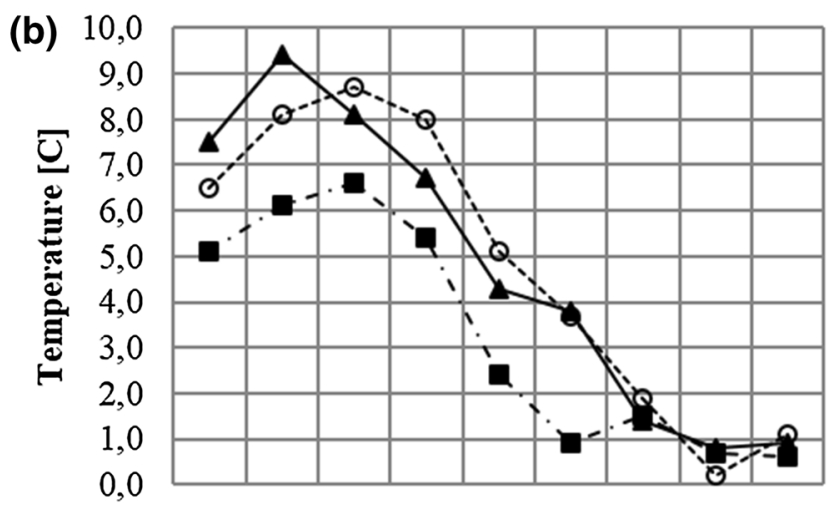

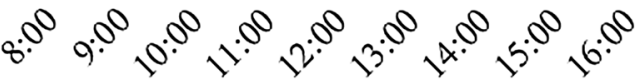

Time [h]

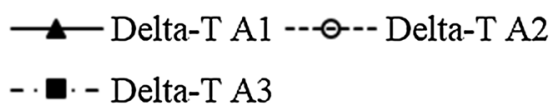

(b) 10,0

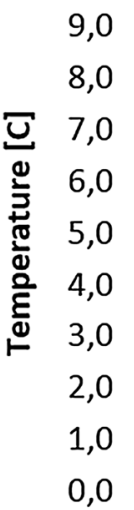

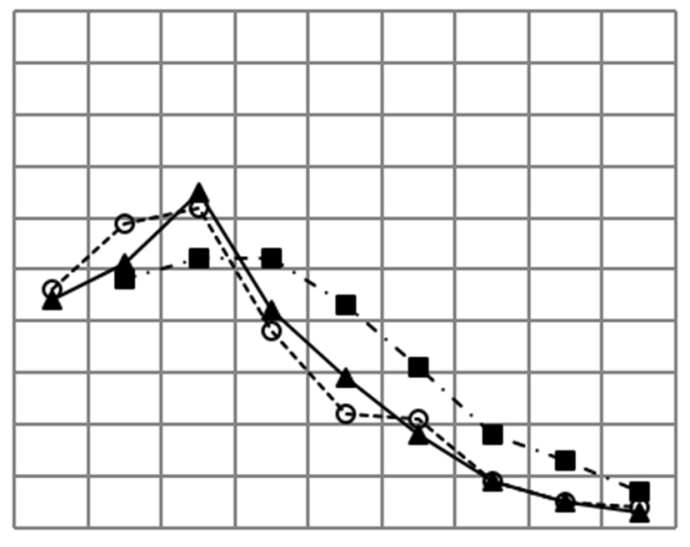

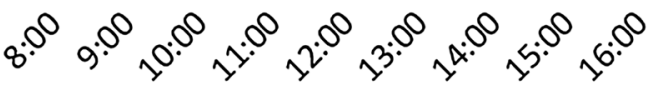

Time [h]

$\longrightarrow$ D Delta-T B1 --@--- Delta-T B2 - - - Delta-T B3

significant, reaching $6.5,6.2$, and $5.2{ }^{\circ} \mathrm{C}$ for $\mathrm{B} 1, \mathrm{~B} 2$ and $\mathrm{B} 3$, respectively. These peak values were observed between 9:00 and 11:00 a.m. It is noteworthy how the system of branched cracks B3 behaved differently, in addition to lower Delta- $\mathrm{T}$, the temperature-dropping rate was slower over time. This result can be attributed to the different type of branched cracking, which had smaller cracks but spread over the study area. This suggests the need to stick to different criteria when studying this type of cracking. 

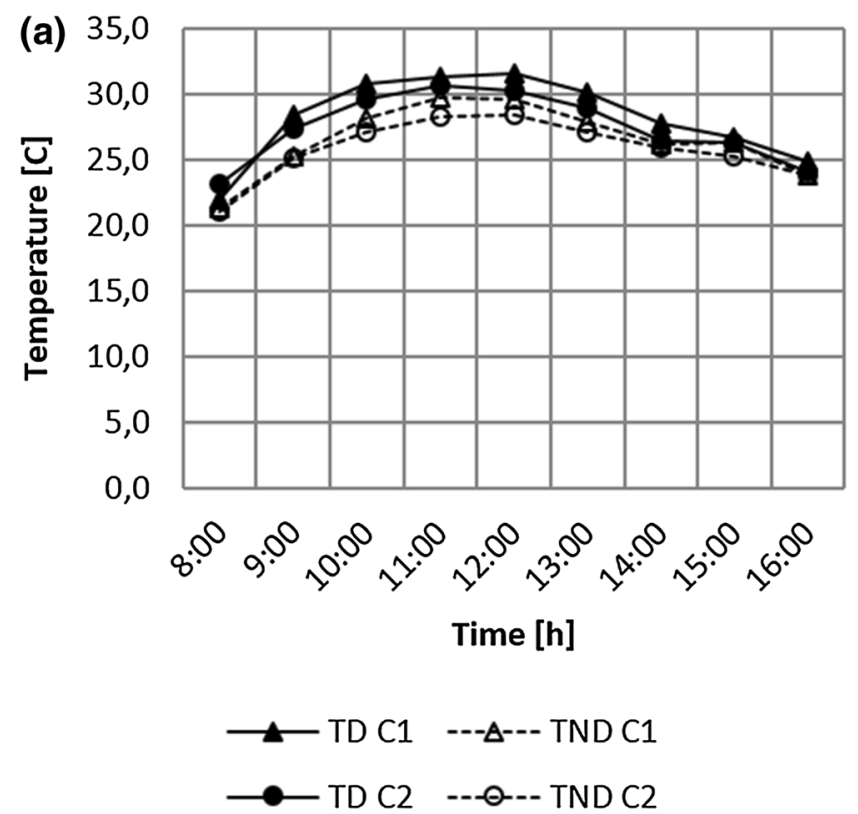

Fig. 6 Region C: a TD and TND evolution; b Delta-T evolution

Figure 6 shows temperature behavior in region C. Two branched cracks were investigated, and TD and TND increased over time, peaking at 12:00 noon. For these cracks, in particular, TD and TND behaved similarly. Thus, even though these cracks are visible in the thermographs (Fig. 3), the Delta-T values were much lower (less than $3{ }^{\circ} \mathrm{C}$ ), indicating that these cracks are more superficial. Moreover, it is important to take into account that the temperature measurement in the thermogram is more difficult in this type of cracking due to its uneven geometry. Again, it points out to the need for more specific criteria for analysis of this type of cracks.

The studies conducted showed that the visual analysis of thermograms (qualitative) provided simple information, which are best understood by quantitative analysis. The anomalies can be classified according to Delta- $T$ results regarding type, and as a preliminary inference regarding severity. Region A had linear cracks and the largest Delta$\mathrm{T}$ values. The thermograms show that the "hottest" area is actually a band along the fissure. This result seems to suggest that perhaps this anomaly has emerged as an initial crack, and as it evolved over time due to the action of rain and temperature, it has propagated even as adhesion failures along the fissure. This is corroborated by the high Delta- $T$ values observed. In region $B$, the crack studied at B1 and B2 spots shows a smaller width band (compared to region A) and lower Delta-T values. Most likely, in these cases, the damage is smaller compared to the previous.

The branched cracks appear sharp in the thermograms. The B 3 crack behaved consistently with the analysis of the region. On the other hand, the $\mathrm{C} 1$ and $\mathrm{C} 2$ cracks had no

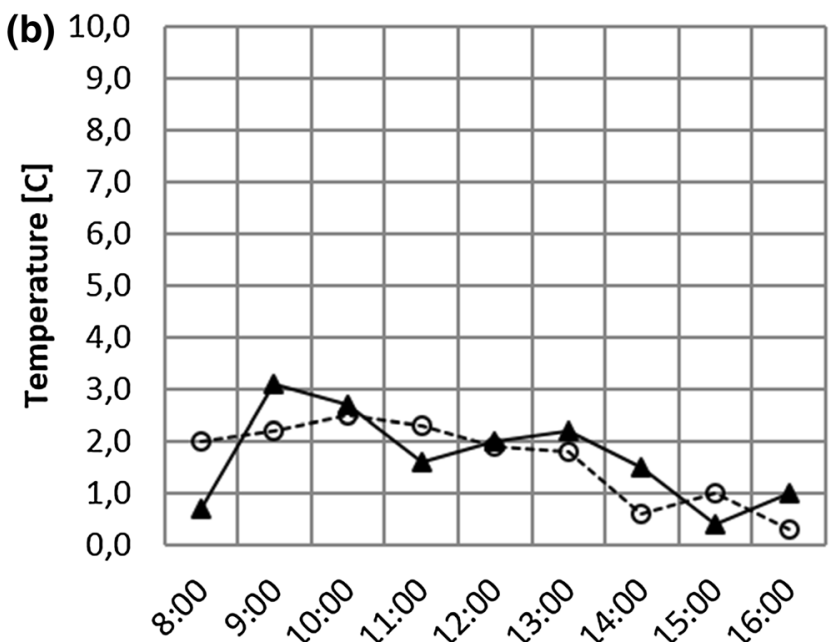

Time [h]

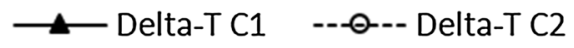

significant Delta-T evolution. Obviously, it raises the question about the possible superficiality of these fissures. However, it is clear that further analysis should be performed. Perhaps defining the degree of branching of the cracks or the use of other parameters, such as the functions of thermal contrast $[4,6,18]$, would allow a more definitive analysis.

\section{Conclusions}

The study performed to assess the behavior of cracks in the facade using the quantitative passive thermography, allowed the main conclusions that are listed below.

The use of passive thermography in the field requires knowing the temperature evolution of the studied element. In this case, the hygrothermal simulation is a very applicable tool.

The maximum temperatures occurred near 12:00 noon, and decreased after that due the lack of sun incidence. Thus, the best period for the thermographic inspection is the morning, when the largest Delta-T values are recorded.

Linear cracks in regions A and B showed the highest Delta-T values, possibly showing associated neighboring degradations.

The branching cracks are difficult to analyze quantitatively, but they can be visually identified in regions with higher Delta-T values. Other approaches are suggested, such as the functions of thermal contrast to better understand and quantify the degree of degradation of elements. 
Field tests are complex, either by the variability of the climate action or by constructive variations and degradation of the studied elements. Thermographic inspection is an auxiliary tool of great application potential, but it must be used with appropriate care by the thermographer, by being careful when taking the pictures and in the subsequent analysis of the thermograms.

Acknowledgments The authors thank the Materials Testing Laboratory of the Universidade de Brasilia for providing the equipment and support to this study, CNPq and CAPES for supporting the research, and L. E. B. Bauer for reviewing and editing the text.

\section{References}

1. Bauer E, Pavon E (2015) Termografia de infravermelho na identificação e avaliação de manifestações patológicas em edifícios. Concreto Construção 79:93-98

2. Barreira E, Freitas VP (2007) Evaluation of building materials using infrared thermography. Constr Build Mater 21:218-224

3. Bauer E, Castro EK, Hildenberg A, Pavon E (2014) Critérios para a aplicação da termografia de infravermelho passiva como tecnica auxiliar ao diagnóstico de patologias em fachadas de edifícios. Rev Politec (Instituto Politec Bahia) 26:266-277

4. Bauer E, Pavón E, Barreira E, Kraus E (2016) Analysis of building facade defects using infrared thermography: laboratory studies. J Build Eng 6:93-104

5. Edis E, Flores-Colen I, Brito J (2012) Passive thermographic inspection of adhered ceramic claddings: limitations and conditioning factors. J Perform Constr Facil. doi:10.1061/(ASCE)CF. 1943-5509.0000365

6. Maldague X (2001) Theory and practice of infrared technology for nondestructive testing. Wiley, NY
7. Paoletti D, Ambrosini D, Sfarra S, Bisegna F (2013) Preventive thermographic diagnosis of historical buildings for consolidation. J Cult Herit 14:116-121

8. Bauer E, Freitas VP, Mustelier N, Barreira E, Freitas SS (2015) Infrared thermography - evaluation of the results reproducibility. Struct Surv 33:20-35

9. Barreira E, Bauer E, Mustelier N, Freitas VP (2015) Measurement of materials emissivity-influence of the procedure. In: 13th Int. Work. Adv. Infrared Technol. Appl., Pisa, Italy

10. ASTM 1933-99 (1999) Standard test methods for measuring and compensating for emissivity using infrared, West Conshohocken, Pennsylvania, United States

11. ASTM E1862-97 (2010) Standard test methods for measuring and compensating for reflected temperature using infrared imaging radiometers, West Conshohocken, Pennsylvania, United States

12. Freitas SS, Freitas VP, Barreira E (2014) Detection of façade plaster detachments using infrared thermography - a nondestructive technique. Constr Build Mater 70:80-87

13. Silvestre JD, de Brito J (2009) Ceramic tiling inspection system. Constr Build Mater 23:653-668

14. Barreira E, Almeida RMSF, Delgado JMPQ (2016) Infrared thermography for assessing moisture related phenomena in building components. Constr Build Mater 110:251-269

15. Edis E, Flores-Colen I, Brito J (2014) Passive thermographic detection of moisture problems in façades with adhered ceramic cladding. Constr Build Mater 51:187-197

16. Edis E, Flores-Colen I, Brito J (2015) Quasi-quantitative infrared thermographic detection of moisture variation in facades with adhered ceramic cladding using principal component analysis. Build Environ 94:97-108

17. Bauer E, Castro EK, Pavon E, Oliveira AHS (2015) Criteria for application and identification of anomalies on the facades of buildings with the use of passive infrared thermography, In: 1st Int. Symp. Build. Pathol., Porto, Portugal, p 12

18. Vavilov V (2014) Noise-limited thermal/infrared nondestructive testing. NDT E Int. 61:16-23 